\title{
Statyba
}

\section{MULTI-PURPOSE SELECTONOVATION IN PREPARATION OF CONSTRUCTIONAL WORK}

\section{E. K. Zavadskas}

To cite this article: E. K. Zavadskas (1995) MULTI-PURPOSE SELECTONOVATION IN PREPARATION OF CONSTRUCTIONAL WORK, Statyba, 1:3, 58-80, DOI:

10.1080/13921525.1995.10531519

To link to this article: https://doi.org/10.1080/13921525.1995.10531519

曲 Published online: 26 Jul 2012.

Submit your article to this journal

山 Article views: 48 


\section{MEHRZIELSELEKTIERUNG BEI DER BAUVORBEREITUNG}

\section{E.K. Zavadskas}

In beliebigen Bereichen der menschlichen Tätigkeit sind bei der Vorbereitung und Ausführung von Produktionsprozessen technische Entscheidungen verschiedener Art zu treffen.

Die Konzeption der Architekten und Konstrukteure wird auf der Grundlage eines allgemeinbaulichen Projektes mit Hilfe technologischer Projektierung und Ausführung technologischer Prozesse verwirklicht. Die Technologie ist ein Zweig der Technik und untersucht den Produktionsprozeß unter zwei Aspekten: den technisch-technologischen (Technologie des Bauprozesses) und organisatorisch-technologischen (Organisation der Technologie).

Jede beliebige technische Entscheidung muß auf der Basis von Analyse und Bewertung folgender Aspekte getroffen werden: dem technisch-technologischen, organisatorischtechnologischen und dem sozialen.

Die technische Entscheidung ist richtig, wenn sie die rationalste ist unter Berücksichtigung aller diesen Aspekte kennzeichenden Hauptfaktoren (Effektivität, lokale Kriterien).

Folglich ist eine komplexe Bewertung aller möglichen Varianten von technishen Entscheidungen anhand einer großen Anzahl verschiedendimensionaler Effektivitätskriterien erforderlich.

In der vorliegenden Arbeit wird ein Aspekt der Technologie - der Technisch-technologische unter Berücksichtigung einiger sozialer Faktoren behandelt.

\section{Schwerpunkte bei der Entscheidungsfindung im Bauwesen}

1. Schwerpunkt: Einflüsse auf Bewertungsprozeß und Bewertungsergebnis (Bild 1)

2. Schwerpunkt: Primärdatenermittlung und -gewinnung

Erfassung, Beschaffung und Aufarbeitung der Ausgangsdaten gehören zu den aufwendigsten Tätigkeiten im Bewertungsproze $\beta$, da sie eine tiefgründige Analyse in allen Produktionsstufen des Bauwesens, umfangreiche Literaturrecherchen und Expertenbefragungen erordern (Bild. 2). 


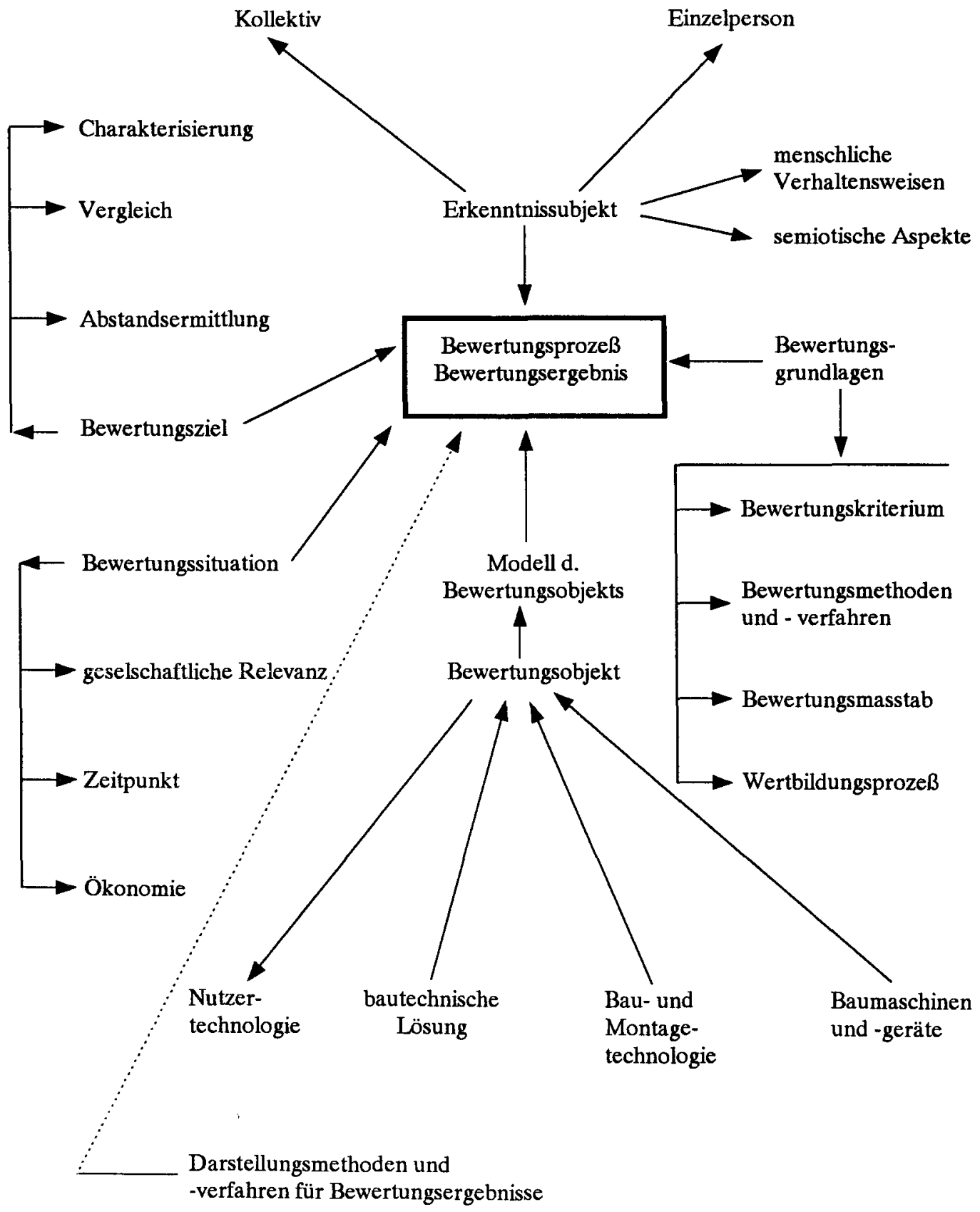

Bild 1. Eiflüsse auf Bewertungsprozeß und Bewertungsergebnis 


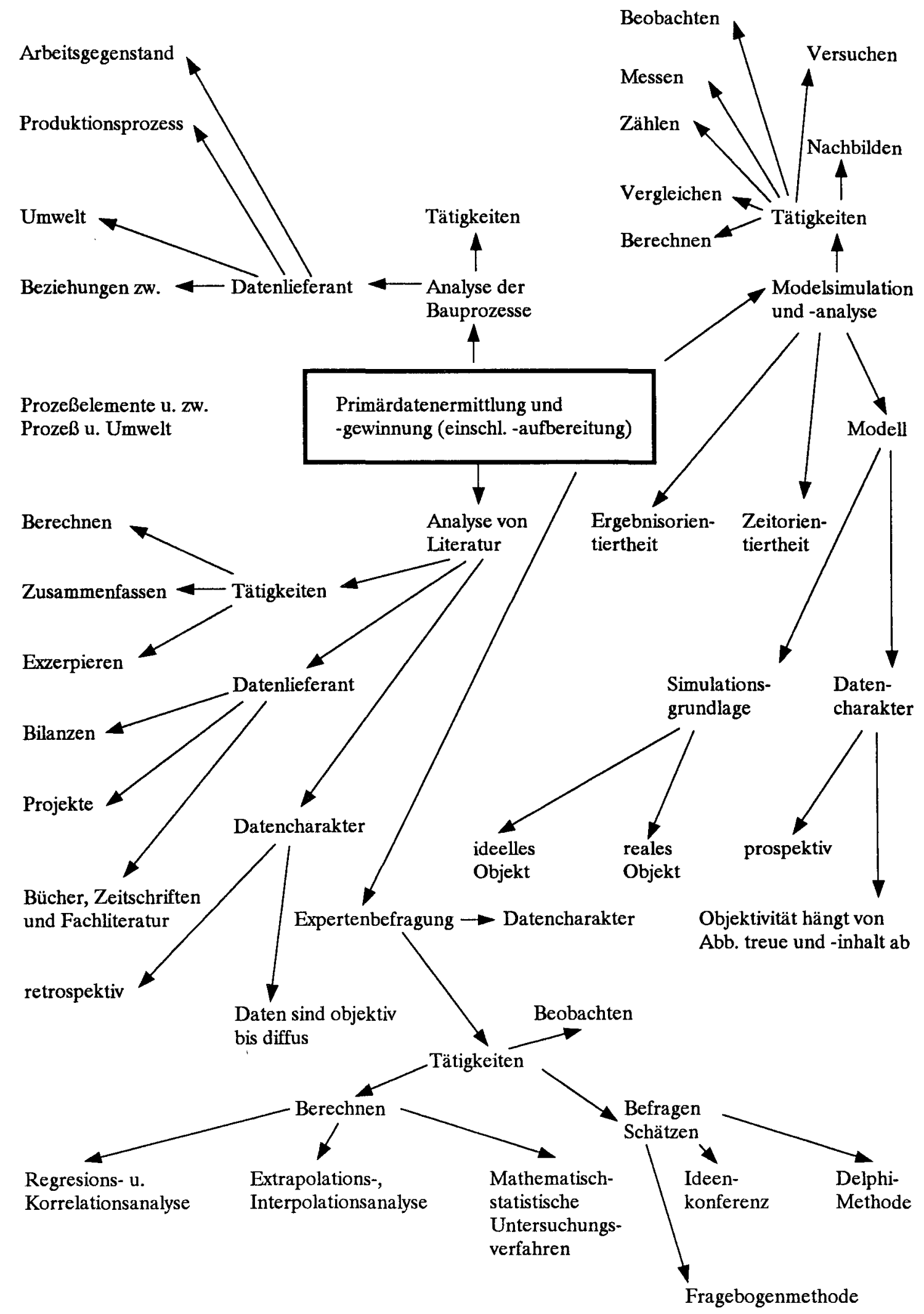

Bild 2. Primärdatenermittlung und -gewinnung 


\section{Schwerpunkt: Methoden zur Bewertung von Lösungen}

Bewertungsmethoden und -verfahren haben den unterschiedlichsten Charakter und Aufbau. Sie reichen von der bewußt subjektiven Einschätzungen bis hin zu objektiven Verfahren auf Basis mathematischer Methoden. Bild 3 zeigt in einer Übersicht Methoden und Verfahren zur Bewertung baulicher Lösungen und zur Wichtung von Effektivitätskriterien auf. Das Bild wird ergänzt mit möglichen Formen der Darstellung von Bewertungsergebnissen.

4. Schwerpunkt: Bestimmung der optimalen Lösungsvariante

Die nach dem Variantengrundsatz erarbeiteten technisch-technologischen Lösungen sind nach folgender Reihenfolge für eine Entscheidung aufzuarbeiten:

1. Übersichtliche Darstellung möglicher Varianten.

2. Aufstellung eines Systems technisch-ökonomischer Bewertungskriterien, einer Bewertungsskala für deren Wichtung sowie Auswahl eines geeigneten Bewertungsverfahrens.

3. Durchführung einer Vorbewertung zum Erhalt der Vorzugsvarianten.

4. Bewertung der verbliebenen Varianten mit Angabe der der optimalen Lösung und Rangfolge der weiteren Lösungen.

In einzelnen wird in Bild. 4 für die Entscheidungsannahme folgender Algorithmus vorgeschlagen.

\section{Hauptsächtliche Prinzipien bei der Entscheidungsfindung im Bauwesen}

Die technische Vorbereitung von Bauproduktion erfordert die Lösung einer ganzen Reihe von spezifischen Aufgaben.

Im Stadium der allgemeinbaulichen Projektierung wählen die Architekten und Konstrukteure eine räumliche und planmäßige Lösung eines Gebäudes oder einer Anlage. Dabei sollten die Möglichkeiten der Technologen zur Verwirklichung der Idee der Projektanten berücksichtigt werden. Die angenommenen Lösungen sollen fertigungsgerecht sein, d. h., bei minimalen Arbeitsaufwand, Bedarf an energetischen und materiellen Ressourcen zu verwirklichen sein. Voraussetzung dafür ist die Kenntnis der modernen Errungeschaften der Bauwissenschaft und technik im Bereich der Technologie der Bauprozesse. Folglich ist die Teilnahme eines Bautechnologen bei einer architektonisch-konstruktionsmäßigen Entscheidung unumgänglich, da sie im wesentlichen Maße die mögliche organisatorisch-technologische Lösung bedingt.

Fertigungsgerechte Projektlösungen können nur im Ergebnis einer Variantenprojektierung auf allen Stadien der technischen Produktionsvorbereitung angenommen werden. 

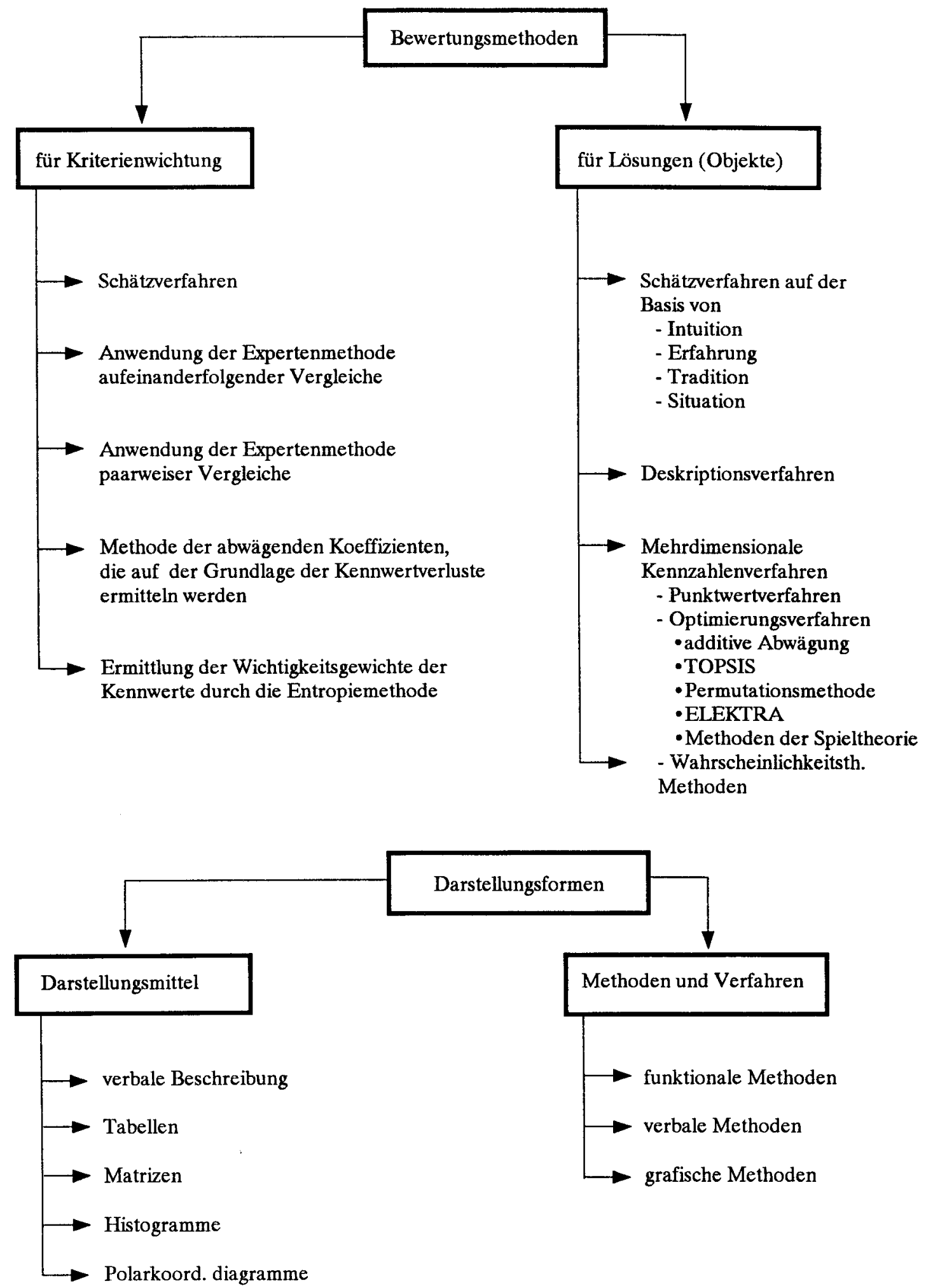

Bild 3. Systematik der Bewertungsmethoden und Darstellungsformen für Bewertungergebnisse 
Unter Ausnutzung von traditionellen Bestimmungsmethoden für die Fertigungsgerechtheit können immer alle spezifischen Besondeiheiten berücksichtigt werden, die unter konkreten Bedingungen der anzunehmenden Entscheidung auftauchen können. Die dabei angenommenen Kennwerte der Effektivität sind nicht immer hinreichend für eine eindeutige Antwort auf die Frage, welche Variante die bessere ist.

Dazu kann bemerkt werden, daß ein objektiveres Ergebnis erhalten wird, wenn die Wahl der Entscheidung durch komplexe Behandlung der architektonischen, konstruktionsmässigen und technologischen und ökonomischen Aforderungen (Faktoren) durchgeführt wird. Dabei steht als Hauptziel die Wahl der besseren Variante bei minimalen Aufwand aller Ressourcenarten.

Die Technologie der Bauproduktion stellt ein System von technischen, organisatorischen und ökonomischen Entscheidungen dar, gerichtet auf die Realisation der architektonischen und konstruktionsmäßigen Konzeptionen, die mit der Projektierung und dem Bau neuer Gebäude und Anlagen, mit der Nutzung neuer Baustoffe und -konstruktionen, mit der Anwendung neuer Organisationsformen der Bauproduktion zusammenhängen.

Anderseits werden die Möglichkeiten der praktischen Verwirklichung der einen oder anderen architektonischen und konstruktionsmäßigen Entscheidung durch technologischen Charakteristiken des Bauvorhabens häufig bedingt und manchmal sogar wesentlich eingeschränkt. Damit erklärt sich, warum es notwendig ist, die Komplexität bei der Ausarbeitung technologischer Entscheidungen durch allseitige Berücksichtigung der Wechselwirkung zwischen den architektonischen und konstruktionsmäßigen Charakteristiken mit der Technologie des Bauens von entsprechenden Gebäuden und Anlagen gewährleisten.

Bei der Verwirklichung der komplexen Behandlung der Bewertung, Prognostizierung und Optimierung architektonischer, konstruktionsmäßiger und technologischer Entscheidung können verschiedentliche mathematische Methoden und Modelle genutzt werden. Die Wahl der Methoden wird hauptsächlich durch die Aufstellung entsprechender Aufgaben und dem Vorhandensein von Ausgangsdaten oder Möglichkeit sie zu erhalten, bestimmt.

Das Problem der Wahl einer Technologievariante für die Errichtung einzelner Konstruktionen kann dann gelöst werdẹ, wenn das Gebäude oder Anlage in technologischer Hinsicht in Teile geteilt werden.

Auf der Grundlage der architektonischen Entscheidung können die bautechnologischen Varianten ausgewählt werden, die die Herstellung der einzelnen Gebäudeteile und Hilfskonstruktionen in einzelnen Etappen des Gebäudebaus wiederspiegeln. Die Wahl der Entscheidung kann unter Berücksichtigung der funktionalen, konstruktionsmäßigen und technologischen Faktoren durchgeführt werden. Dabei müssen alle zu dem errichtenden Gebäude gehörenden technologischen Varianten untereinander abgestimmt, in Gruppen zusammengefaßt und verglichen werden. 


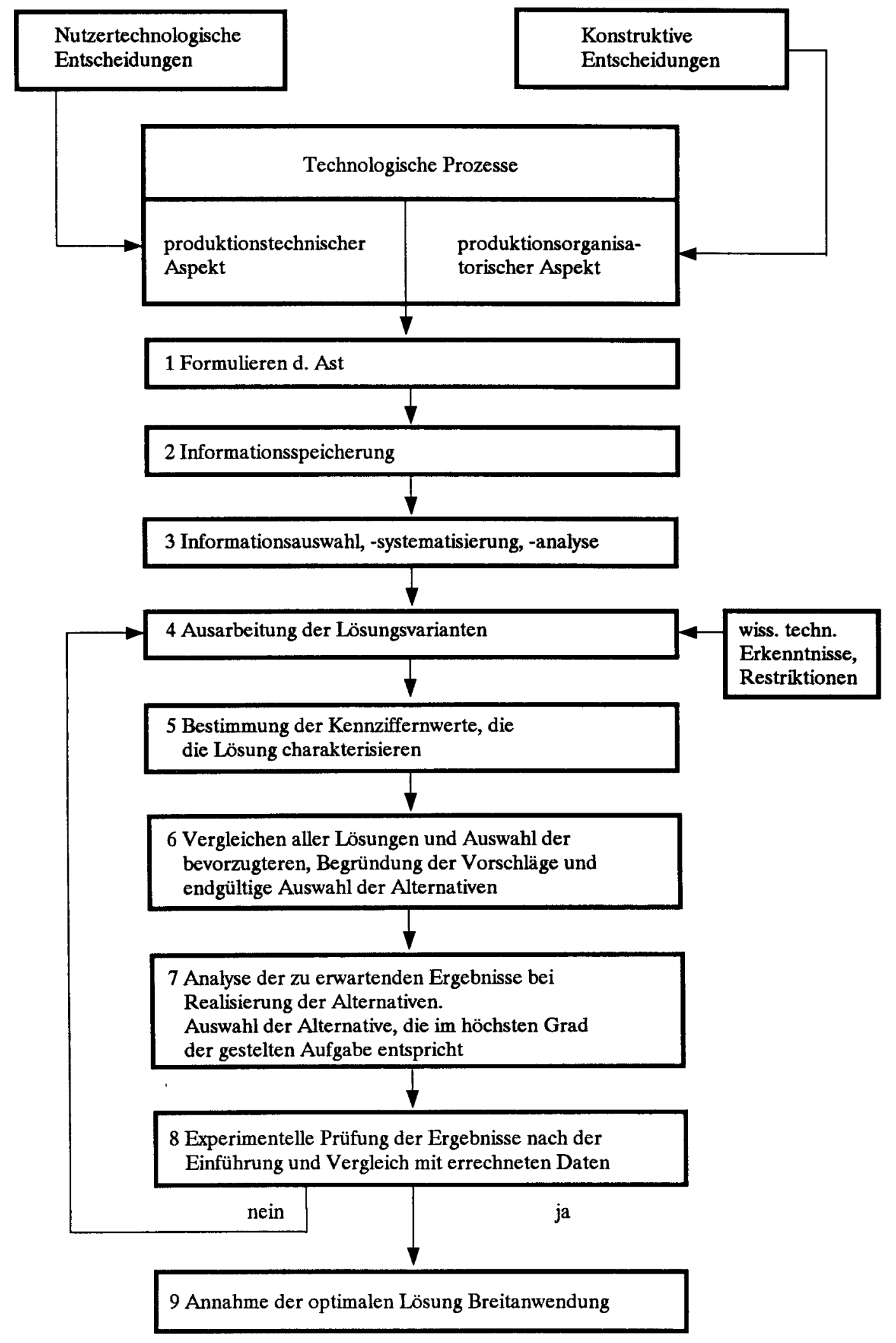

Bild 4. Entwicklung optimaler technisch-technologischer Lösungen (Arbeitsschritte) 
Die Art der Effektivitätskennziffern und Vergleichenskennziffern muß in Abhängigkeit von der Art der zu lössenden Aufgabe festgelegt werden.

Die Wichtigkeit der Effektivitätskennziffern sollte in Abhängigkeit von der Art der zu lösenden Aufgabe festgelegt werden.

Bei der Begründung der Effektivitätskennziffern ist zu beachten, daß folgende Faktoren den Prozeß der Entscheidungsannahme beeinflussen können:

- Ausgangswerte (Parameter der Anlagen, z. B. Errichtung von Fundamenten, geologische und hydrogeologische Charakteristiken);

- bauliche technich-technologische Einschränkungen (z. B. Einschränkungen in bezug auf die Konstruktionselastizität, Tiefe der Baugrube);

- betriebliche Einschränkungen (z. B. die zu untersuchende Variante ist nicht ausführbar, da bei der vorhandenen technologischen Ausrüstung die Genauigkeit der Herstellung ungenügend ist und keine Möglichkeit zur Verbesserung der Ausrüstung oder Kooperation der Arbeiten besteht);

- Einschränkungen, die für die zu projektierende Technologie spezifisch sind (für die zu wählende Methode);

- Einschränkungen, die entscheidende Bedeutung haben (z. B. der Bau einer Anlage in extrem kurzer Zeit mit zusätzlichen Kosten für die Lösung der vorgeschlagenen Problematik);

- Standardseitige Einschränkungen (z. B. begrentzter Bauplatz bei Rekonstruktionen).

Zur Zeit ist ohne Analyse und mehrkriterielle Entscheidung in der Tat eine hohe Technologie und die Ressourcensparung in der Bauproduktion nicht möglich. Deshalb haben die Erarbeitung und die praktische Anwendung der Methoden der mehrkriteriellen Entscheidung bei der Vorbereitung der Bauproduktion eine besonders große Bedeutung.

Um eine begründete effektive Entscheidung zu erhalten, ist eine eindeutige Aufgabestellung, Formulierung, Zusammenfassung und Bearbeitung von Expertenbewertung und Ausnutzung von wissenschaftlich motivierten Methoden bei Anwendung von Mensch-Maschine-Verfahren erforderlich, die erlauben, aus der gesamten Untermenge die zulässigen Varianten einer Variantenanzahl auszuwählen, die in einem bestimmten Sinne der vorgegebenen bevorzugten am nächsten ist.

Unter dem Begriff "in großeren Maße bevorrzugt" versteht man das Vorhandensein von nicht nur einem Kennwert der Effektivität, der für die Bewertung der Technologie ausgenutzt wird, sondern die Gesamtheit von Kennziffern. Jede der möglichen Varianten muß nach allen Kennziffern bewertet werden und auf Grund dieser Bewertung soll die Entscheidung angenommen werden, ob eine Variante auch weiter untersucht wird oder weggelassen werden 
kann. Dafür muß ein Verfahren erarbeitet werden, daß eine volle Durchsicht aller möglichen Varianten gewährleistet.

Auf diese Weise kann die Aufgabe in Form folgender Teilaufgaben dargestellt werden, deren erfolgreiche Lösung erlaubt, die erste Etappe der Wahl optimalen Technologiewarianten zu formulieren:

- Erarbeitung eines Verfahrens zur Darstellung der Technologiewarianten, geeignet für die volle Übersicht aller Varianten der Ausgangsmenge;

- Erarbeitung eines vollen Satzes Effektivitätskennziffern, sowie einer Bewertungsskala für jede Kennziffer der Effektivität und ein Bewertungsverfahren;

- Aufbau formaler Verfahren, die erlauben, auf der Grundlage einer Untermenge der stärker Bevorzugung von Alternativen auszustellen;

- Entwicklung eines formalen Verfahrens, das ermöglicht, auf der Grundlage der bevorzugteren Varianten eine Bevorzugungsreihe der Alternativen aufzubauen.

Die ersten zwei Aufgaben sind Vorbereitungsetappen für die Lösung der dritten und vierten Aufgabe, die als eine der Aufgabenstellung der Theorie der Meinungsannahme angesehen werden kann, und zwar als Vielkriterienaufgabe der Entscheidungsannahme.

Die Kompliziertheit der mehrkriteriellen Entscheidung besteht in ersten Linie in dem Widerspruch der Kriterien. Es ist notwendig, ein gewisses Schema der rationellen Kompromisses anzuwenden, der die harmonische Verbesserung der Qualität der zu treffenden Vorausentscheidung nach allen besonderen Optimalitätskriterien erlaubt, die auf Grund mehrerer besonderer Optimalitätskriterien $x_{1}, x_{2}, \ldots, x_{j}, \ldots, x_{n}(x \in X, \forall j, j=\overline{1, n})$ die ein Vektorkriterium $x=\left(x_{1}, x_{2}, \ldots, x_{j}, \ldots, x_{n}\right)$ darstellen, eingeschätzt werden.

Jede Kennziffer charakterisiert eine der Eigenschaften der Alternative. Beim Vergleichen der Alternativen (Varianten, Projekte) ist es zweckmäßig, sowohl die Kennziffern, deren Wert verbessert, als auch die Kennziffern mit sich verschlechterndem Wert zu berücksichtigen. Dabei dürfen nur dann bestimmte Kennziffern unbeachten bleiben, wenn man vollkommen davon überzeugt ist, daß der Wert derselben für alle Alternativen gleichartig ist.

Der Zahlenwert der Kennziffern, die für die Charakteristik der Bauvarianten benutzt werden, hängt von einer großen Anzahl zufälliger Faktoren ab: von natürlichen, technischen, sozialen, politischen und dlg., die die Umstände oder das äußerliche Milieu formieren, in dem die Maßnahmen realisiert werden. Wenn diese Faktoren die für die Charakteristik der Alternativen angenommenen Kennziffern nicht wesentlich beeinflussen, so wird die Entscheidung für ganz bestimmte (determinierte) Bedingungen getroffen. 
Im entgegensetzten Fall wird die Entscheidung unter Berücksichtigung der unbestimmten Umstände erarbeitet. Manchmal unterliegt der Einfluß der zufälligen Faktoren auf die Ergebnisse bekannten Gesetzmäsigkeiten, dann ist es möglich, die Wahrscheinlichkeit zu errechnen zum Erhalt von Ergebnissen, die nicht unter einem bestimmten Niveau liegen. In solchen Situationen sagt man, daß die Entscheidung unter Risikobedingungen begründet wird.

Die Kennziffern werden in äussere, technisch-ökonomische und technisch-unterhaltungsbedingte, und innere, konstruktions-technologische und organisatorische klassifiziert.

Die Berechnung mehrdimensionaler Effektivitätskennziffern erfolgt auf der Grunde Normen. Für nicht genormte Kennziffern werden Hinweise für die Berechnung gegeben und Analogien gesucht.

In jeder beliebiger Situation werden die Alternativen durch die Gesamtheit der Kennziffern charakterisiert. Um die Alternativen zu vergleichen und die beste zu wählen, müssen die Vor- und Nachteile jeder Alternative objektiv bewertet werden. Eine solche Bewertung kann entweder direkt anhand der Gesamtzeit der die Alternative kennzeichnenden Kennziffern oder nach einem speziellen Merkmal - dem Kriterium, das auf der Basis der Gesamtheit der Werte dieser Kennziffern formiert wurde, vorgenommen werden.

Unbestimmtheit, d. h. unvollständige oder ungenauere Vorstellung über die Größen der einzelnen Parameter in der Zukunft, wird durch verschiedene Ursachen hervorgerufen. Man kann bedingt zwei Arten von Unbestimmtheit herausstellen:

- Unbestimmtheit, als Folge unvollständiger Kenntniss der Gesetzmäßigkeiten;

- Unbestimmtheit, verursacht durch Zufall.

Auf Bild 5 sind eine bedingte Aufteilung der Unbestimmtheiten nach Arten und Methoden zur Berücksichtigung bei Annahme von technischen oder organisatorisch-technologischen Entscheidungen gegeben.

Die Auswahl eines konkreten Modells und einer Regel, nach der die Entscheidungssuche erfolgt (Entscheidungsregel), hängt vom Charakter des Problems und der erarbeitenden Entscheidung, sowie, in gewissem Maße auch vom Charakter der Alternative ab. Die Alternativen können einander ausschließend, kombinierbar sein, diskreten oder kontinuierlichen Charakter tragen.

Nehmen wir einen allgemeinen Fall, wenn $\mathbf{n}$ Alternativen vorhanden sind, die nach $\mathrm{m}$ Kennziffern verglichen werden. Es ist vicht schwer, jede Alternative nach jeder konkreten Kennziffern zu bewerten. Weitaus kompliziert ist es, die Alternativen nach verschiedenen Kennziffern zu vergleichen. Die Kennziffern haben verschiedene Dimensionen oder sind aus anderen Beweggründen schwer vergleichbar. Deshalb ist es bequemer, die Alternative $A_{j}$ und Ergebnisse ihrer Realisation $a_{i j}$ in Matrixform darzustellen. 


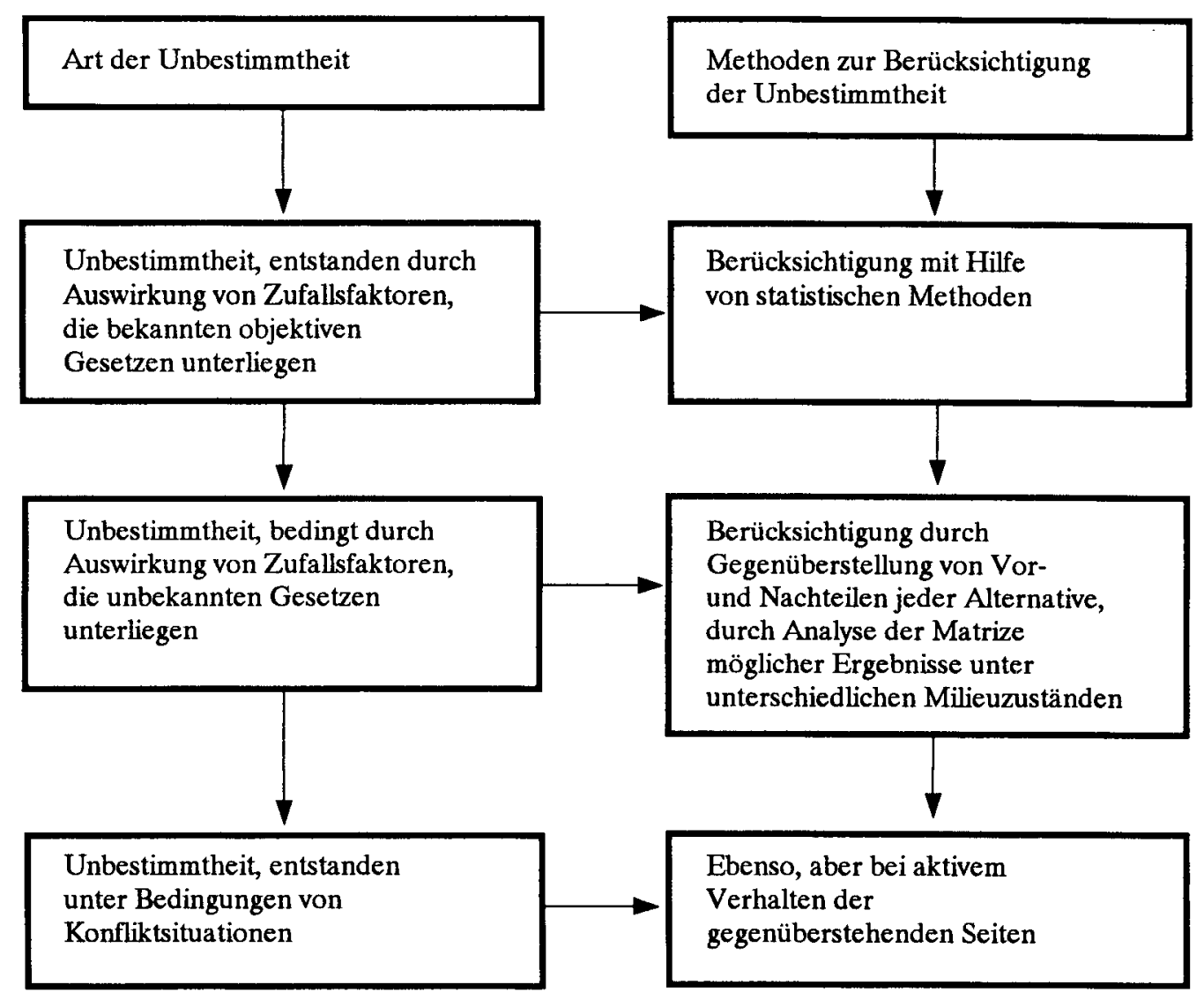

Bild 5. Bedingte Teilung der Unbestimmtheit nach Arten

Man nehme an, es gibt vier Alternativen $A_{1}, A_{2}, A_{3}, A_{4}$ und vier Kennwerte zu ihrer Bewertung.

Mann stellt eine Ergebnismatrix auf:

\begin{tabular}{|c|c|c|c|c|}
\hline \multirow{2}{*}{ Alternativen } & \multicolumn{4}{|c|}{ Kennwerte - Nr. } \\
\cline { 2 - 5 } & 1 & 2 & 3 & 4 \\
\hline$A_{1}$ & $a_{11}$ & $a_{12}$ & $a_{13}$ & $a_{14}$ \\
\hline$A_{2}$ & $a_{21}$ & $a_{22}$ & $a_{23}$ & $a_{24}$ \\
\hline$A_{3}$ & $a_{31}$ & $a_{32}$ & $a_{33}$ & $a_{34}$ \\
\hline$A_{4}$ & $a_{41}$ & $a_{42}$ & $a_{43}$ & $a_{44}$ \\
\hline
\end{tabular}

Jedes Ergebniss stellt eine gewisse Menge von Folgerungen der Realisation konkreten Alternativen dar. Im allgemeinen Fall kann diese Menge aus verschiedensten Kennwerten und Größen bestehen. Für die Alternative $A_{1}$ gibt es ein Ergebnissvektor: 


$$
A_{i}=/ a_{i 1}, a_{i 2}, \ldots, a_{i j}, \ldots, a_{i m} /
$$

Sehr selten kann die Ergebnismatrix für die Auswahl der besten Alternative genutzt werden. Zum Beispiel, wenn eine der Alternative in jedem der Kennwerte jede andere Alternative übertrifft. Gewöhnlich aber ist es erforderlich, Ergebnissen vershiedener Qualität eine einheitliche Bewertung zu geben, um sie dann vergleichen zu können und die beste Alternative $\mathrm{zu}$ wählen. Dafür ist jedes Ergebnis mit einer dimensionslosen Bewertung $r_{i j}$ zu vergleichen, um somit einen Bewertungswektor zu erhalten:

$$
A_{i}=/ r_{i 1}, r_{i 2}, \ldots, r_{i j}, \ldots, r_{i m} /
$$

Die Größen $r_{i j}$ werden als Nutzeffekt der $i$-ten Alternative laut $j$-ter Kennziffer bestimmt. Sie soll dimensionslos sein, damit man verschiedene Nutzeffekte oder Nutzgrößen quantitativ vergleichen könnte.

Daraus folgt, daß, wenn in der Matrix der zu erwartenden Ergebnisse von der Realisation der Alternativen die Grösen $a_{i 1}, \ldots, a_{i m}$ irgendwelche Qualitätskennwerte darstellen (z. B. Kosten, Arbeitsaufwand, Sicherheit, Festigkeit, Festigungsgerechtheit, Verbrauch wertvollen Materials usw.), so sind es im Bewertungsvektor, $r_{i 1}, \ldots, r_{i m}$ - Ziffern.

Analog zu den Ergebnissmatrizen stellt man eine Bewertungsmatrix auf:

\begin{tabular}{|c|c|c|c|c|}
\hline \multirow{2}{*}{ Alternativen } & \multicolumn{4}{|c|}{ Kennwerte - Nr. } \\
\cline { 2 - 5 } & 1 & 2 & 3 & 4 \\
\hline$A_{1}$ & $r_{11}$ & $r_{12}$ & $r_{13}$ & $r_{14}$ \\
\hline$A_{2}$ & $r_{21}$ & $r_{22}$ & $r_{23}$ & $r_{24}$ \\
\hline$A_{3}$ & $r_{31}$ & $r_{32}$ & $r_{33}$ & $r_{34}$ \\
\hline$A_{4}$ & $r_{41}$ & $r_{42}$ & $r_{43}$ & $r_{44}$ \\
\hline
\end{tabular}

Die weitere Aufgabe läuft auf die Gegenüberstellung der Bewertungsvektoren verschiedener Alternativen hinaus. Eine solche Aufgabe ist ebenfalls kompliziert. Zur Vereinfachung der Gegenüberstellung einzelner Alternativen ist empfohlen, alle Menge ist günstig von Kennwerten im Form von drei ihrer Rolle nach verschiedener Gruppen oder Untermengen der Kennwerte darzustellen (Bild 6).

Zur ersten sind alle Kennwertforderungen (Untermengen $y_{j}$ ) zu zählen, d. h. solche Kennwerte, bei deren Nichtübereinstimmung die Entscheidungswariante abgelennt wird. Zur zweiten Gruppe gehören die Bewertungskennwerte (Untermenge $w_{j}$ ), nach denen die Gegenüberstellung der Entscheidungsvarianten erfolgt und in die dritte Gruppe kommen die Bedingungen (Untermengen $z_{j}$ ), die berücksichtigt werden müssen. 
Matrix der

Ausgangskennwerte

(Ergebnisse)

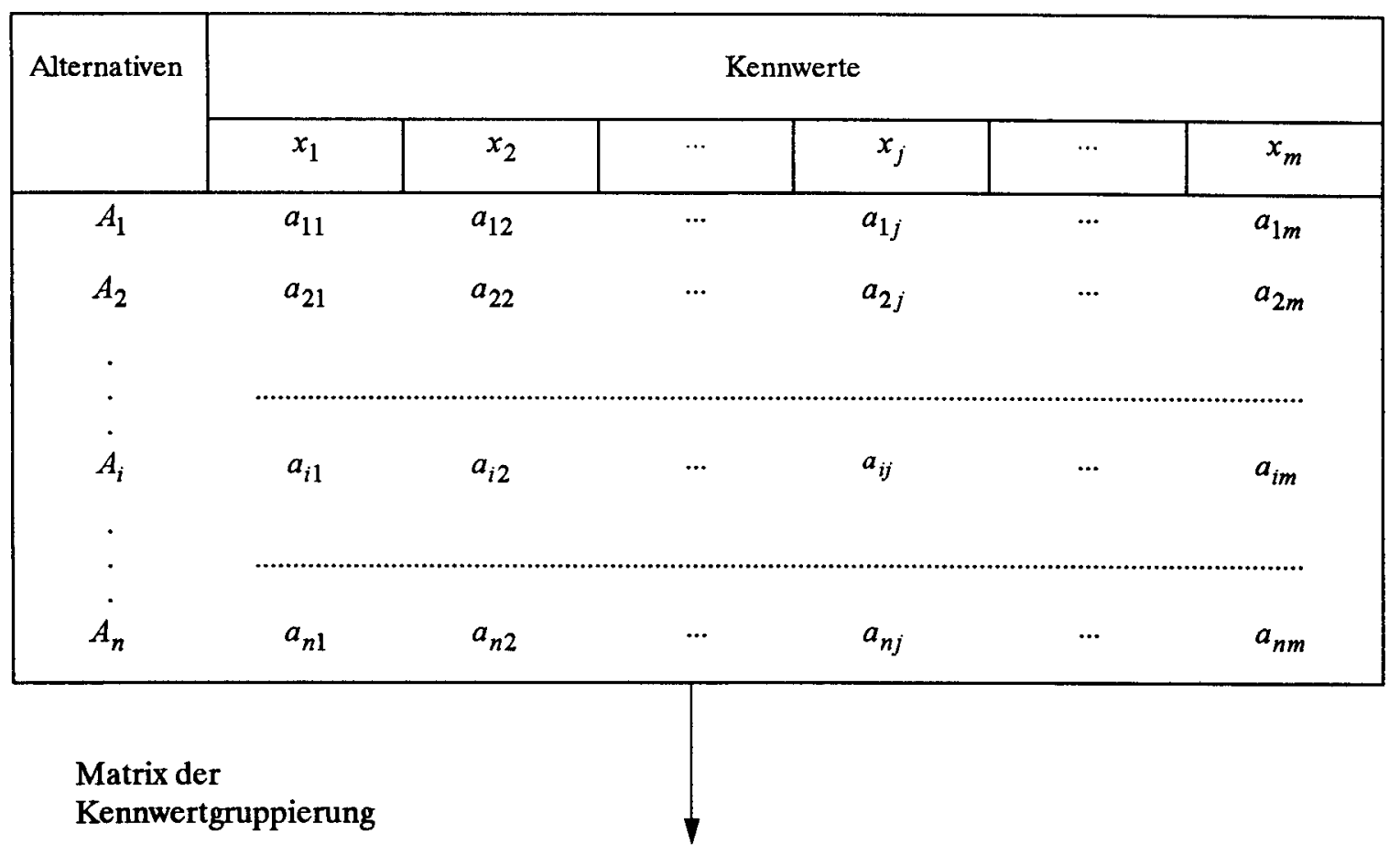

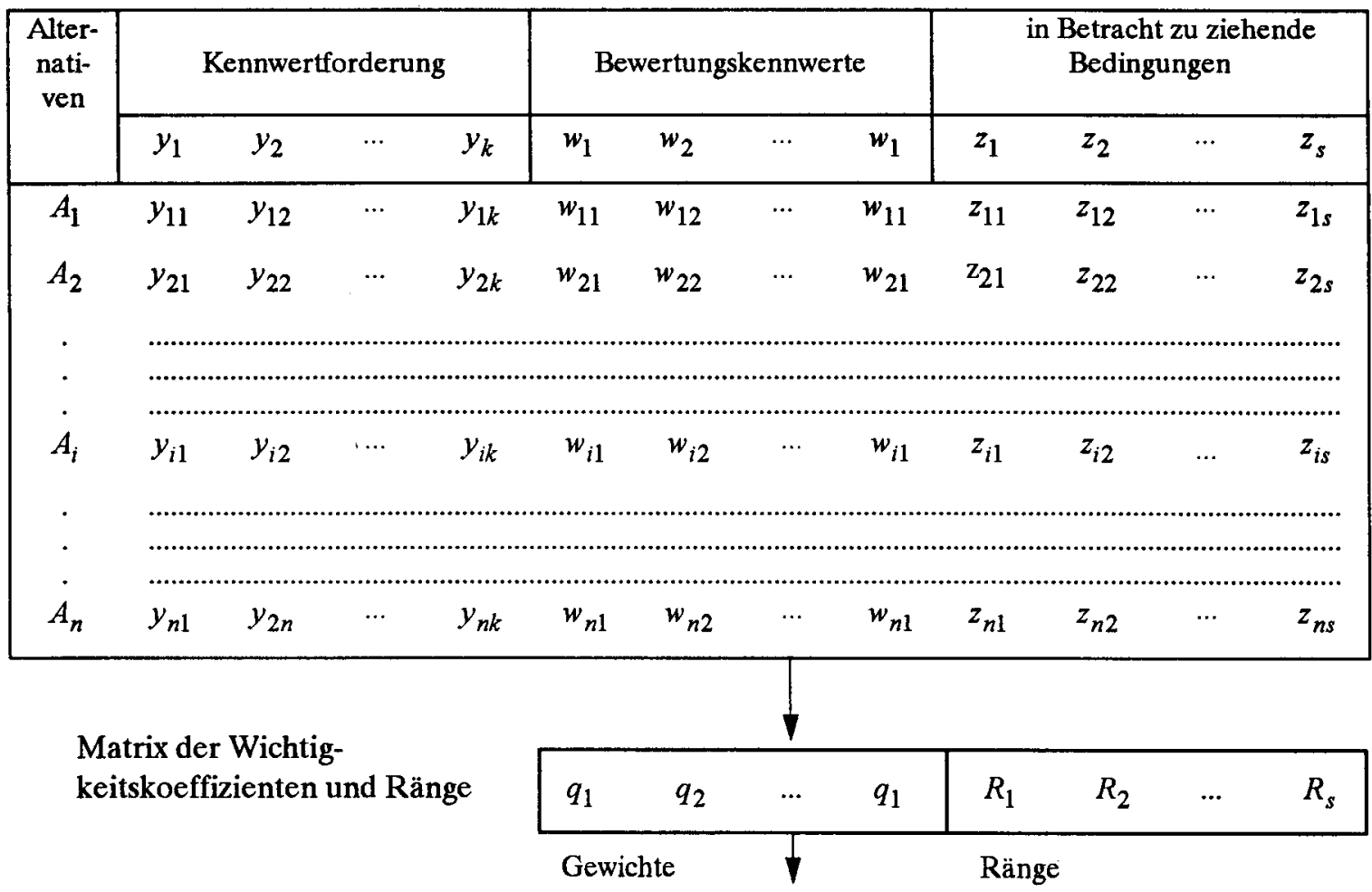


Matrix der transformierten

Kennwerte (Bewertungsmatrixe)

\begin{tabular}{|c|c|c|c|c|c|c|c|c|}
\hline \multirow{2}{*}{$\begin{array}{l}\text { Alterna- } \\
\text { tiven }\end{array}$} & \multicolumn{4}{|c|}{ Bewertungskennwerte } & \multicolumn{4}{|c|}{ in Betracht zu ziehende Bedingungen } \\
\hline & $w_{1}$ & $w_{2}$ & $\ldots$ & $w_{1}$ & $z_{1}$ & $z_{2}$ & $\ldots$ & $z_{s}$ \\
\hline$A_{1}$ & $w_{11}$ & $w_{12}$ & $\cdots$ & $w_{11}$ & $z_{11}$ & $z_{12}$ & $\cdots$ & $z_{1 s}$ \\
\hline$A_{2}$ & $w_{21}$ & $w_{22}$ & $\cdots$ & $w_{21}$ & $z_{21}$ & $z_{22}$ & $\cdots$ & $z_{2 s}$ \\
\hline$\cdot$ & ........ & 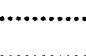 & . & ….... & (n).......... & 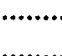 & 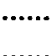 & (........... \\
\hline . & (n..... & 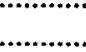 & $\cdots \cdots$ & ......... & 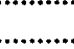 & ............. & ......... & 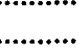 \\
\hline$A_{i}$ & $w_{i 1}$ & $w_{i 2}$ & $\cdots$ & $w_{i 1}$ & $z_{i 1}$ & $z_{i 2}$ & $\cdots$ & $z_{i s}$ \\
\hline . & $\cdots \cdots$ & & 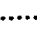 & ........ & & & 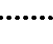 & (............... \\
\hline . & ....... & $\ldots$ & $\cdots$ & ........ & $\ldots \ldots$ & $\ldots \ldots$ & ....... & ............ \\
\hline$A_{n}$ & $w_{n 1}$ & $w_{n 2}$ & $\cdots$ & $w_{n 1}$ & $z_{n 1}$ & $z_{n 2}$ & $\cdots$ & $z_{n s}$ \\
\hline
\end{tabular}

Matrix der Kriterien

\begin{tabular}{|c|c|c|c|c|}
\hline \multirow[t]{2}{*}{ Alternativen } & \multicolumn{4}{|c|}{ Kriterien } \\
\hline & $K_{1}$ & $K_{2}$ & $\ldots$ & $K_{k}$ \\
\hline$\overline{A_{1}}$ & $k_{11}$ & $k_{12}$ & $\ldots$ & $k_{1 k}$ \\
\hline$A_{2}$ & $k_{21}$ & $k_{22}$ & $\ldots$ & $k_{2 k}$ \\
\hline . & - & . & $\ldots$ & . \\
\hline - & · & · & $\cdots$ & - \\
\hline$A_{i}$ & $k_{i 1}$ & $k_{i 2}$ & $\ldots$ & $\dot{k_{i k}}$ \\
\hline . & - & . & $\cdots$ & . \\
\hline . & . & . & $\begin{array}{l}\ldots \\
\ldots\end{array}$ & . \\
\hline$A_{n}$ & $k_{n 1}$ & $k_{n 2}$ & $\ldots$ & $k_{n k}$ \\
\hline
\end{tabular}

\begin{tabular}{|c|c|}
\hline Alternativen & Nutzenswerte (Bevorzugungen) \\
\hline$A_{1}$ & $N_{1}$ \\
$A_{2}$ & $N_{2}$ \\
$\cdot$ & $\cdot$ \\
$\cdot$ & $\cdot$ \\
$A_{i}$ & $\cdot$ \\
$\cdot$ & \\
$A_{n}$ & $-N_{i}$ Auswahl des optimalen Wertes \\
\hline
\end{tabular}

Bild 6. Schema der Alternativeauswahl 
Für die Untermenge der Kennwerte werden die Bedeutungskoeffizienten ermittelt, die den Wichtungsgrad jeder Kennziffer festlegen. Die in Betracht zu ziehenden Bedingungen können rangmäßig nach Bevorzugung aufgeteilt werden. Anschliessend werden die Alternativen nach dem Grad der Übereinstimmung mit diesen Kennziffern bewertet.

Es ist natürlich, daß bei einer solchen Vielfältigkeit der zu bewertenden Kennwerte mit verschiedenem Sinn und unterschiedlichen Dimensionen bei der Überfürung der Kennwerte aus der Ergebnissmatrix in die Kennwerte der Bewertungsmatrix Schwierigkeiten auftreten. Es ist durchaus ersichtlich, daß eine unmittelbare Gegenüberstellung der Ergebnisvektoren infolge ihrer Polidimensionalität nicht immer möglich ist.

In der allgemeinen Theorie der Annahme von Entscheidungen ist eine große Anzahl von Methoden zur Auswahl der Entscheidung für die Ermittlung der bevorzugten Alternative entwickelt worden. Die Anwendung einer oder anderer Methode wird durch Inhalt der zu lösenden technischen Aufgabe bedingt. Deshalb sollte die Entscheidung treffende Person (ETP) die Regel bestimmen, nach der die Auswahl erfolgen soll und erst danach zur Anwendung einer oder anderer dem Charakter des zu lösenden Problems entsprechenden formalen Methode übergehen.

Deshalb ist bei der Lösung vershiedener Probleme ein differenziertes Herangehen notwendig, was eine lokale interpretation erfordert. Die Auswahl der Alternativen soll nach Methoden erfolgen, die den Hauptaxiomen der Theorie der Entscheidungsannahme entsprechen.

Bei der Wahl der Alternativen können Aufgaben mit vershiedenem Charakter gelöst werden. So wurde schon anfänglich von der Vielfältigkeit der möglichen Alternativen nach ihrem Charakter gesprochen. Die Aufgaben der Alternativwahl können mit direkter Formulierung oder eben aus dem aus der Endmenge bekannten abgeleitet werden. Es existiert eine große Anzahl von Methoden zur Erstellung der Ordnung der Bevorzugung der Alternativen, die in der Fachliteratur beschrieben wurden.

Nach Erfassung der Wichtigkeitskoeffizienten $q_{j}$ und der Ränge $R_{j}$ erhält man eine transformierte Kennwertmatrix, in der dimensionslose Effektivitätskennwerte erfaßt sind. Nach diesen Kennwerten, unter Ausnutzung einer oder einiger Methoden der Theorie der Entscheidungsannahme, werden die Effektivitätskriterien jeder der Alternativen $i$ nach dem Kriterium $j\left(K_{i j}\right)$ ermittelt und Matrix $K$ aufgestellt. Wenn die Wahl der besten Alternative nach einem Effektivitätskennwert ausgefürt wird, so erhält man die Vektorspalte der Größen. Die Alternative wird nach dem besten Wert des Kriteriums $K_{i}$ ausgewählt. Wenn die Wahl der besten Alternative nach einigen Regeln verwirklicht wird, entsprechend auch nach verschiedenen Effektivitätskriterien, so erhält man die Einheitsmatrix der Kriterien $K_{i j}$.

Bei Vorhandensein von mehrerer Effektivitätskriterien, wählt die ETP die beste Alternative aufgrund der Mehrzahl (positiver vom Standpunkt des erwünschten Ergebnisses) der Kriterien, 
die zu der zu wählenden Alternative gehören. Im gegebenen Falle können ebenfalls einige Methoden der Bevorzugungsbewertung ode: anderer Effektivitätskriterien angewendet werden.

Aufgrund der Bewertung der Effektivitätskennwerte wird die Vektorspalte der Nutzensbewerbung der Alternativen $\mathrm{N}$ oder die Skala ihrer Bevorzugung aufgebaut.

Dazu muß bemerkt werden, daß auf dem Bild 6. ein verallgemeinertes Schema der Entscheidungsannahme angegeben ist. Wenn konkrete Methoden der Theorie der Entscheidungsannahme genutzt werden, so kann die Reihenvolge der Berechnungen etwas geändert werden. Einige der durchzuführende Schritte der Berechnung können ausgelassen oder andere zusätzllich eingeführt werden.

\section{Bewertungsmethoden der Mehrfaktorenalternativen}

\subsection{Klassifizierung}

In dieser Klassifikation werden nur Methoden der Vektoroptimierung behandelt, die bei der Wahl der besten Alternative aus ihrer Endmenge verwendet werden. Dabei setzt man voraus, daß es sich um Mehrfaktorenalternativen handelt, d. h. daß sie durch einen Satz (Vektor) verschiedendimensionaler Kennwerte ihrer Effektivität beschrieben werden. Die Klassifikation stützt sich auf den Charakter der Information, die die Entscheidung treffende Person (ETP) benötigt.

1. Methoden, die keine zusätzliche Information erfordert (maximin, Dominanz und andere);

2. Methoden die die Angabe von Information bezüglich der Kennwerte erfordern, die die zu vergleichenden Alternativen charakterisiert.

2.1. Methoden, bei denen zusätzliche Information genutzt werden, die kardinalen (numerischen) Charakter tragen (Methode der linearen Aufgaben, Methode der einfachen additiven Abwägung, Methode der hierarchischen additiven Abwägung, Methode Elektra und andere);

2.2. Methoden, in denen genügend Information von ordinalem (Ordnungs-) Charakter gegeben sind (lexikographische Methode, Eliminierung nach Aspekten, Methode der Permutation);

2.3. Methoden, die Kenntnisse der Grenznormen der Kennwertsubstitution erfordern (Methode der hierarchischen Wechselwirkung);

3. Methoden, in denen das Vorhandensein von Information in bezug auf die Alternative vorausgesetzt wird.

3.1. Methoden, in denen Information über paarweise Vergleiche der Alternativen genutzt werden (LINMAP, interaktive Methode der einfachen additiven Abwägung); 
3.2. Methoden, bei denen das Vorhandensein von Information über die Ordnung der paarweisen Ähnlichkeit vorausgesetzt wird (Methode der Mehrfaktorenskalierung mit idealem Punkt).

Der Sinn der Bewertung und Auswahl von Mehrfaktorenalternativen liegt in der Verarbeitung der anfänglichen Information in Form von polydimensionalen Effektivitätskennwerten, die in der Beschreibung der Alternativen derart enthalten ist, daß es möglich wird, eine endgültige Auswahl der besten Alternative zu treffen oder eine Reihe Bevorzugung aufzubauen.

Man kann zwei Hauptverfahren bei der Organisation solch einer Verarbeitung herausstellen Modelle ohne und mit Substitution (dementsprechend ohne oder mit Kompensation).

In den Modellen ohne Substitution kann Verlust in einem Kennwert nicht durch Gewinn in einem anderen ausgeglichen werden. Jeder Kennwert wird in diesem Falle als ein vollkommen einzelner aufgefaßt.

Die Modelle für die Auswahl von Mehrfaktorenalternativen ohne Kompensation sind meistens sehr einfach und schränken die Möglichkeiten der ETP äußerst ein. Zu diesen Modellen gehören auch die Methoden des Maximin, die lexikographischen Methoden und andere.

In den Modellen mit Substitution ist der Verlust in einem Kennwert durch den Gewinn in einem anderen zulässig. Eine solche Substitution ist sowohl in sehr begrenzten, als auch in weiten Bereichen möglich. Das hängt vom Charakter der zu lösenden Aufgaben und von der ETP ab.

Dabei ist fastzustellen, daß bei Bewertung der Alternativen nach Modellen ohne Kompensation die Alternativen nach jedem Kennwert zu vergleichen sind, während nach Modellen mit Kompensation meistens jeder Mehrfaktorenalternative eine einzige Zahl zugeordnet wird, auf deren Grundlage der Vergleich und die endgültige Wahl der Alternative getroffen wird.

In Abhängigkeit davon, auf welche Prinzipien sich der Prozeß der Bestimmung der obengenannten Zahl stützt, können die Modelle mit Kompensation in drei Hauptgruppen eingeteilt werden:

1. Modelle, in denen die Alternative gewählt wird, die den größten Nutzen hat. Bei diesen Modellen besteht das Hauptproblem darin, wie die geeignete Nutzensfunktion zu bestimmen ist.

Zu dieser Gruppe gehören folgende Methoden: die Methode der einfachen additiven Abwägung, die Methode der hierarchischen additiven Abwägung.

2. Modelle, bei denen die idealen Alternative möglichst nahe Alternative gewählt wird. Das sind die sogenannten Kompromißmodelle, zu denen die Methoden LINMAP und TOPSIS zu zählen sind.

3. Modelle der Koordinierung. In diesen Modellen wird eine Menge von Bevorzugungsbeziehungen vorgegeben, die die größten Maßgrößen der Übereinstimmung haben. $\mathrm{Zu}$ dieser Gruppe gehören Methoden der Permutation, der linearen Bestimmung, Methode ELEKTRA. 


\section{Mehrzielselektierung technologischer Entscheidungen der Bauvorbereitung}

Die Varianten nach den Prinzipien und wissenschaftlichen Grundlagen der Systemtechnik unter Benutzung der Methoden der mehrkriteriellen Optimierung sind einzuschätzen. Die Entscheidungen für Bauwerke und Anlagen insgesamt werden an Hand der systemtechnischen Einschätzung der Entwurfsvarianten verwirklicht. Die Mehrzielauswahl, die Wahl der technologischen und ressourcensparenden Entscheidungen, die zielstrebige Vervollkommnung in verschiedenen Etappen der Vorbereitung der Bauproduktion behandelt die spezielle Wissenschaftsrichtung - Mehrzielselektierung technologischer Entscheidungen der Bauvorbereitung (von. Lat. selectio - die Auswahl).

Die Effektivität der zu fällenden technischen und organisatorisch-technologischen Entscheidungen hängt im hohem Grade von anzuwendenden Methoden der systemtechnischen Einschätzung, den Entscheidungsvarianten und der Mittel ihrer Steuerung ab.

Der Prozeß der Lenkung der Einschätzung der Entwurfslösungen ist stadienartig und enthält fünf Stufen der mehrkriteriellen Entscheidung.

In der Stufe 1 werden die Anforderungen an die Qualität des Ergebnisses gebildet, das nach der Realisierung der Entwurfslösung erzielt wird. In dieser Stufe der mehrkriteriellen Entscheidung gehen allgemein die Prozesse der wissenschaftlichen Forschung, der Versuchs- und Konstruktionsarbeiten, der technisch-ökonomischen Begründung, der Projektierung von Bauvorhaben, der Expertise der Entwurfs- und Kostenunterlage, der Vorbereitung der Bauproduktion ein. Einige von ihnen können nicht in die erste Etappe der mehrkriteriellen Entscheidung eingehen.

Das hängt von dem sozialem Auftrag zur Erarbeitung des Projektes ab. Bei der Anwendung der bekannten Technologien und der konstruktiven Lösungen können die Etappen der wissenchaftlichen Forschung, der Versuchs- und Konstruktionsarbeiten sowie einige andere fehlen.

Grundlage der 1. Etappe der mehrkriteriellen Entscheidung sind das Projekt des zu erarbeitenden Vorhabens und die organisatorisch-technologischen Unterlagen. Die Hauptziele der Etappe 1 der mehrkriteriellen Entscheidung sind die Auswahl der optimalen (rationellen) Entscheidung im Stadium der Projektierung (Optimierungsweg), die Auswahl der zu treffenden Entscheidung einiger im Voraus festgelegten Forderungen - die Prüfung im Stadium der Expertise (Standardweg). Die Etappe 1 der mehrkriteriellen Entscheidung ist die herrschende bezüglich der anderen, weil in dieser Etappe das NIveau der künftigen Technologie, ihre Neuheit, Effektivität, Perspektivität, Arbeits-, finanziele, materielle, energetische und andere Aufwände bestimmt werden. 
Das Ziel der Etappe 2 der mehrkriteriellen Entscheidung sind die Entscheidungen für die Produktionsdurchführung in den Betrieben der Bauindustrie. Dabei ist die Einhaltung der im Entwurf vorgesehenen Forderungen der Technologie obligatorisch. Folglich sind die Eingänge der Etappe 2 der mehrkriteriellen Entscheidung - die Ergebnisse der Etappe 1 der mehrkriteriellen Entscheidung, die die Technologie der Betriebe der Bauindustrie betreffen. Als gesteuerte Variablen der Etappe 2 der mehrkriteriellen Entscheidung sind das Vorhaben der Produktion (AM), die Informationsressourcen und die Arbeitskräfte (AK). Ausgang der Etappe 2 der mehrkriteriellen Entscheidung ist die Fertigproduktion, die den Forderungen des Entwurfes entspricht und für die Anwendung tauglich ist.

Die Etappe 3 der mehrkriteriellen Entscheidung ist der technologischen Kompletierung, dem Transport und der Lagerung der nötigen Ressourcen und den Arbeitsmitteln für die Bauvorhaben gewidmet. Als Eingang der Etappe 3 dient die Information, die von Etappe 2 und 1 über die Eigenschaften der hergestellten Produktion und ihrer notwendigen Menge für die Erfüllung der Etappe 3 der mehrkriteriellen Entscheidung (an Hand der Ergebnisse der Etappe 1) kommt. Ausgang der Etappe 3 ist die Gewährleistung der Etappe 4 mit allen notwendigen Ressourcen.

Die Etappe 4 dient zur Realisierung der durch die Etappe 1 vorgesehenen technologischen Entscheidungen auf der Baustelle. Als Eingang der Etappe 4 ist die Information über die Forderungen dieser Etappe (auf Grund der Ergebnisse der Etappe 1), als Ausgang der Etappe 4 ist die beendete Bauproduktion, die die in Etappe 1 projektierten Anforderungen erfüllt.

Die Etappe 5 der mehrkriteriellen Entscheidung wird im Stadium der Nutzung der Bauproduktion erfült und ist auf die Suche und Realisierung der rationellen Betriebsarten der Technologie bei der Nutzung der einzelnen Konstruktionsteile (der Dachhaut, der Fasadenoberfläche usw.) und der Gebäude und Anlagen insgesamt gerichtet. In diesem Fall ist es die Periodizität der Reparatur einzelner Konstruktionsteile, die Termine der laufenden Generalreparaturen, die Vorausschauung der Möglichkeit (der Notwendigkeit) der Rekonstruktion. Eingang dieser Etappe sind besondere Optimalitätskriterien, die sich als Ergebniss der Realisierung der 4 Etappe ergeben. Ausgang ist die Bauproduktion, deren Qualität sich im Laufe der Zeit auf den von der 1 Etappe vorgesehenen Wert ändert.

Alle Etape sind in je fünf Kreise der Steuerung eingeschlossen. Ihre gesamte Zahl beträgt 15 (siehe Bild 7). Der Operator der Optimierung für mehrkriteriellen Entscheidung ist die laufende Verbindung - transitive binäre Verbindung, die die Anordnung der Elemente der Menge der möglichen Lösungen in eine Reihe nach einer gewissen Regel erlaubt, d. h. die Ordnung der Elemente der Menge $x(a)$ nach einem gewissen Optimalitätsprinzip. Als Ergebnis der Realisierung der Modelle der mehrkriteriellen Entscheidung gelingt es, eine rationelle Lösung $a^{\circ}$ oder eine rationelle Untermenge $A^{\circ}$ zu finden. Die Modelle der mehrkriteriellen Entscheidung können durch analytische oder heuristische Methoden verwirklicht werden, mit oder ohne 
Anwendung der EDFA. Dabei des Kompromisses oder als Gebiet der optimalen Lösungen die Untermenge $A^{\circ}$ aus der Menge $A$ definiert, die sich dadurch unterscheidet, daß jede Lösung $a \in A^{\circ}$ ohne Verminderung des Niveaus für mindestens ein Optimalitätskriterium nicht verbessert werden kann. Daraus folgt, ist $a^{1} \in A$, so erhält man für den Fall von zwei besonderen Optimalitätskriterien $x_{1}(a)>x_{2}\left(a^{1}\right), x_{2}(a)<x_{2}\left(a^{1}\right)$ oder umgekehrt, d.h. für beliebige zwei Lösungen aus $A^{\circ}$ bekommen wir die Wiedersprüchlichkeit der besonderen Optimalitätskriterien und die Entscheidung ist nur an Hand eines Kompromisses möglich. Auf diese Weise gilt für alle Probleme der mehrkriteriellen Entscheidung das Modell

$$
A^{\circ}=\left\{a / a \in A, \quad\left\{a^{1} / x_{1}\left(a^{1}\right) \geq x(a)\right\} \cap A \neq \varnothing\right\}
$$

für alle Optimalitätskriterien

$$
x^{\circ}=\left\{x / x \in X, \quad\left\{x^{1} / x^{1} \geq x\right\} \cap X \neq \varnothing\right\} .
$$

In den Praxis reicht oft die Bestimmung des Bereiches des Kompromisses der technischen und der organisatorisch-technologischen Entscheidungen nicht aus. Es ist notwendig, eine oder mehrere konkurierende Entscheidungen zu finden. Ihre Wahl und der Qualitätsvergleich im Bereich des Kompromisses und des ihm entsprechenden Optimalitätsprinzips möglich. Die Regelung des Raumes der besonderen Optimalitätskriterien und der Wahl der rationallen Entscheidungen wird auf Grund von besonderen Axsiomen durchgeführt. Wenn die Axsiome formuliert sind, wird das Problem der mehrkriteriellen Entscheidung streng formalisiert, und die Wahl der rationellen Lösung zu einer technische Aufgabe, die mit EDVA zu lösen ist.

Es wurden die Grundlagen der mehrkriteriellen Entscheidung formuliert und das universelle automatisierte System der komplexe Einschätzung und der Wahl der ressourcensparenden Bauentscheidungen erarbeitet.

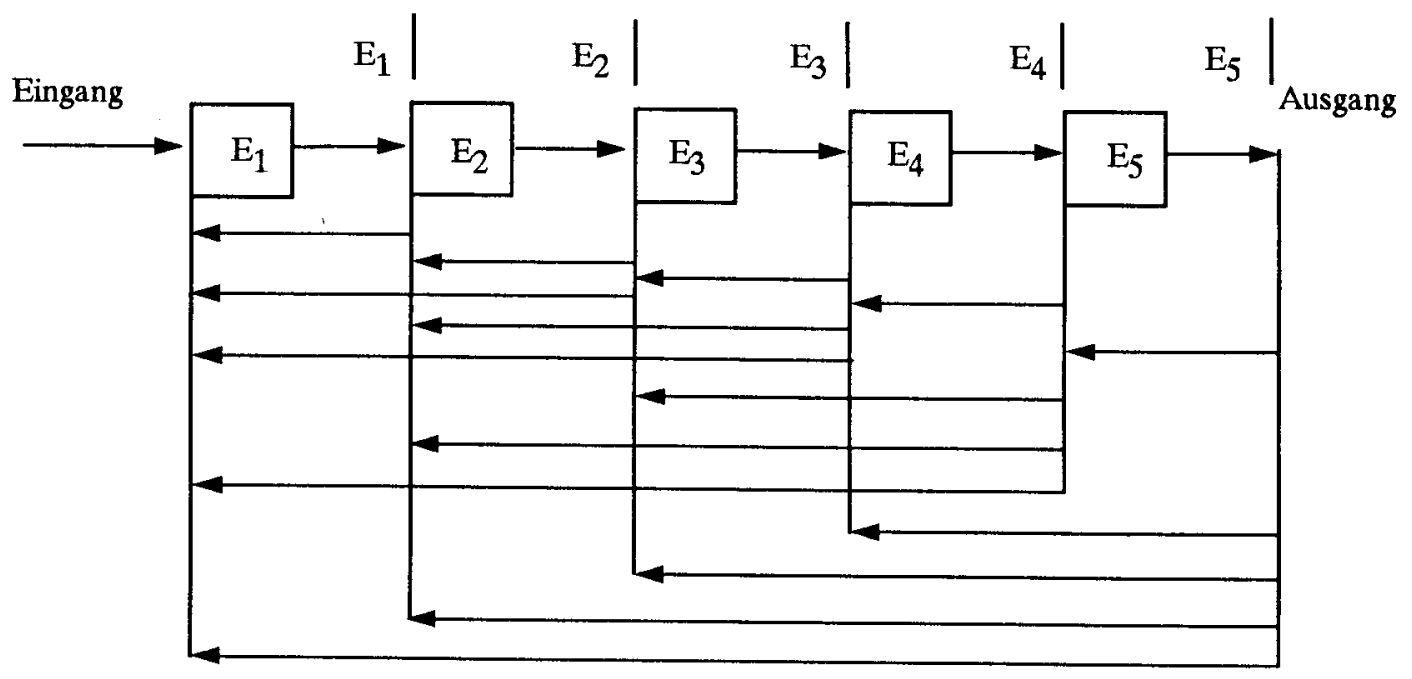

Bild 7. Lokale Etapen der mehrkriteriellen Entscheidung des Bauprozesses 


\section{Systeme der Entscheidungsfindung}

Das universalle automatisierte System umfaßt einen Komplex von Methoden und Vorgängen der mehrkriteriellen Optimierung, die die Entscheidungen für die Bedingungen der vollen Bestimmtheit, der stochastischen Unbestimmtheit und vollen Unbestimmtheit der Ausgangsinformation zu treffen erlauben. Die volle Darstellung der erarbeiten Methoden ist in [1-10] angeführt. Mit dem Einsatz des erarbeiteten universellen automatisierten Systems der Entscheidungen wird der Aufbau der Reihe der Bevorzugung der Varianten und die Auswahl der möglichst rationellsten verwirklicht.

In Zusammenarbeit mit Vilniusser technisches Universität (VTU) und TH Leipzig wird ein Programmsystem Entscheidungsfindung entwickelt, das dem Nutzer ermöglichen soll, mehrkriterielle Entscheidungsprobleme verschiedenster Art zu lösen [8].

Unten Anwendung der Prinzipien und der Methoden der mehrkriteriellen Entscheidung wurden viele Systeme der Entscheidungsfindung geschafen, die völlig mit der Ausgangsinformation versorgt sind.

\section{Schlußfolgerungen}

Die entwickelten Methoden der mehrkriteriellen Entscheidung sind einfacher und im Vergleich weniger arbeitsaufwendig als die in der letzten Zeit auf verschiedenen Gebieten der Technik der Funktions-Kosten-Analyse angewendeten, deren Nutzung im Unterschied zu den vogeschlagenen Methoden der mehrkriteriellen Entscheidung die Kenntnis einer größeren Zahl von Abhängigkeiten zwischen den einzelnen Kennzahlen des zu erarbeitenden technischen Systems verlangt. Deshalb kann man sie hauptsächtlicht für die Erhöhung der Qualität und die Verminderung des Arbeitsaufwandes relativ einfacher technischer Erzeugnisse anwenden.

Bei der mehrkriteriellen Entscheidung für technische und organisatorisch-technologische Varianten treffen wir im Bauwesen am häufigsten drei Typen von Problemen: die diskrete Auswahl auf Grund der verschiedendimensionalen besonderen Optimalitätskriterien, die Wahl der Parameterwerte der zu treffenden Entscheidungen und die Synthese der technologischen Lösungen.

Die Einschätzung der Variantenauswahl mit Methoden der mehrkriteriellen Entscheidung entspricht dem Optimierungsprinzip und berücksichtigt die ständige Vervollkommnung der technologischen Lösungen auf Grund des Komplexes der besonderen Optimalitätskriterien, die möglichtvöllig ihre Effektivität kennzeichnen. 


\section{Literatur}

1. E.Zavadskas. Komplexe Bewertung und Auswahl ressourcensparender Entscheidungen im Bauwesen. Vilnius: Mokslas, 1987, 209 S.

2. E.Zavadskas, A.Kaklauskas. Automated Multivariant Design of Buildings, Multi-purpose Comprehensive Evaluation and Selection of the most Efficient Versions. Aalborg: Aalborg Universitetscenter, $1991,66 \mathrm{p}$.

3. E.Zavadskas, E.Bejder, A.Kaklauskas. Multiple Criteria Analysis of Projects. Aalborg: Aalborg universitetscenter, 1992, $93 \mathrm{p}$.

4. K.Fiedler, F.Peldschus, E.Zavadskas. Methoden der bautechnologischen Entscheidung // Wiss. Berichte TH Leipzig, H.17, 1986, 56 S.

5. E.Zavadskas, F.Peldschus, A.Kaklauskas. Multiple Criteria Evaluation of Projects in Construction / Institute of Technological and Ekonomical Development (ITED). Vilnius Technical University. Vilnius: Technika, 1994, 226 p.

6. E.Zavadskas. Variantenauswahl mit der Nutzensfunktion // Wiss. Zeitschrift der TH Leipzig. Jg 14. H 5/6, 1990, S. 263-273.

7. R.Seeling, E.Zavadskas. Mehrzielselektierung bei technologischen Entscheidungen der Bauvorbereitung // IX internationaler Kongress industrieles Bauen (IKIB). Leipzig: TH Leipzig, Wiss. Berichte, H.5, 1991, S.170-179.

8. E.Zavadskas, I.Löhne, F.Peldschus. LEVI - ein Programm zur Lösung von mehrkriteriellen Entscheidungsaufgaben //VTU mokslo darbai: Statybos technologija ir menedžmentas. T.7. Vilnius: Technika, 1993, p. 8-14.

9. E.Zavadskas. Komplexe Bewertung und Auswahl ressourcensparender Entscheidungen im Bauwesen - 12 internationäler Kongress über Anwendungen der Mathematik in der Ingenieurwissenschaften //Wiss. Zeitschrifft der Hochschule für Architektur und Bauwesen Weimar, H.3. Weimar, 1990, S.95-97.

10. Ch.Wagner, E.Zavadskas, L.Rupprecht. Ergebnisse der Zusammenarbeit der Wissenschaftsbereiche Technologie der HAB und des VISI // Wiss. Zeitschrift HAB Weimar. Jg 31. H. 3B, 1985. 1985, S. 91-93.

\section{STATYBOS GAMYBOS DAUGIATIKSLE் SELEKTONOVACIJA}

\section{E. K.Zavadskas}

S a n tra a k a

Straipsnyje analizuojamos pagrindinès sprendimų prièmimo statyboje problemos, pateikiamos schemos, charakterizuojančios sprendimų priẻmimo etapus. Analizuojami pagrindiniai sprendimų prièmimo principai, formalizuota bendra sprendimy prièmimo eiga. Atlikta daugiafaktorinių alternatyvu ivertinimo klasifikacija. Aptariami daugiakriterijinès selektonovacijos penki etapai bei suformuluotas daugiakriterijiniu sprendimy matematinis modelis. 


\section{MULTI-PURPOSE SELECTONOVATION IN PREPARATION OF CONSTRUCTIONAL WORK}

\section{E.K.Zavadskas}

$\mathrm{Sum}$ m a ry

The article deals with the major problems of decision making in building construction. The schemes describing stages of decision making are presented. The main principles of decision making in building construction are discussed and the sequence of decision-making processes is generalized. An evaluation classification of multi-factorial alternatives is worked out. Five stages of multiple criteria selectonovations in building construction are characterized. The article also presents a mathematical model of multiple criteria solutions. 